\title{
Aldosterone directly affects apelin expression and secretion in adipocytes
}

\author{
He Jiang ${ }^{1, *}$, Xiao-Ping $\mathrm{Ye}^{1, *}$, Zhong-Yin Yang ${ }^{2, *}$, Ming Zhan', Hai-Ning Wang', \\ Huang-Min Cao', Hui-Jun Xie', Chun-Ming Pan', Huai-Dong Song' \\ and Shuang-Xia Zhao'
}

${ }^{1}$ State Key Laboratory of Medical Genomics, Shanghai Institute of Endocrinology and Metabolism, Molecular Medicine Center, ${ }^{2}$ Department of General Surgery, Shanghai Institute of Digestive Surgery, Ruijin Hospital, Shanghai Jiaotong University School of Medicine, Shanghai 200025, China

*(H Jiang, X-P Ye and Z-Y Yang contributed equally to this work)
Correspondence should be addressed to S-X Zhao Email zhaozhao1215@gmail.com

\begin{abstract}
There is a high incidence of metabolic syndrome among patients with primary aldosteronism (PA), which has recently been associated with an unfavorable cardiometabolic profile. However, the underlying mechanisms have not been clarified in detail. Characterizing aldosterone (Ald) target genes in adipocytes will help us to elucidate the deleterious effects associated with excess Ald. Apelin, a novel adipokine, exerts beneficial effects on obesityassociated disorders and cardiovascular homeostasis. The objective of this study was to investigate the effects of high Ald levels on apelin expression and secretion and the underlying mechanisms involved in adipocytes. In vivo, a single-dose Ald injection acutely decreased apelin serum levels and adipose tissue apelin production, which demonstrates a clear inverse relationship between the levels of plasma Ald and plasma apelin. Experiments using 3T3-L1 adipocytes showed that Ald decreased apelin expression and secretion in a time- and dose-dependent manner. This effect was reversed by glucocorticoid receptor (GR) antagonists or GR (NR3C1) knockdown; furthermore, putative HREs were identified in the apelin promoter. Subsequently, we verified that both glucocorticoids and mineralocorticoids regulated apelin expression through GR activation, although no synergistic effect was observed. Additionally, detailed potential mechanisms involved a p38 MAPK signaling pathway. In conclusion, our findings strengthen the fact that there is a direct interaction between Ald and apelin in adipocytes, which has important implications for hyperaldosteronism or PA-associated cardiometabolic syndrome and hoists apelin on the list of potent therapeutic targets for PA.
\end{abstract}
Key Words
- aldosterone
- apelin
- 3T3-L1 adipocyte
- glucocorticoid receptor
- p38 MAPK

\author{
Journal of Molecular \\ Endocrinology \\ (2013) 51, 37-48
}

\section{Introduction}

Aldosterone (Ald), the most potent mineralocorticoid secreted by the adrenal cortex, was originally described as an important regulator of blood pressure and electrolytic balance (Williams 2005). Emerging evidence has shifted the focus to the crucial role of excess Ald in the pathogenesis of cardiovascular events and metabolic syndrome (MS) (Fallo et al. 2006). It is well known that obesity is frequently accompanied by increased plasma 
Ald levels, which are correlated with the amount of adipose tissue (Ehrhart-Bornstein et al. 2004). When chronically exposed to excess Ald, adipose tissue, especially visceral fat, may display disturbed pro- and anti-inflammatory adipokine profiles. There is evidence of a significant correlation between excess Ald and altered serum adipokine levels. Patients with primary aldosteronism (PA) present with reduced leptin and adiponectin levels, as well as increased resistin levels (Torpy et al. 1999, Fallo et al. 2007, Iacobellis et al. 2010). Furthermore, the serum levels of other cytokines, such as osteopontin, interleukin 6 (IL6), TGF $\beta 1$ (TGFB1), and TNF $\alpha$, were perturbed in PA patients (Irita et al. 2006, Carvajal et al. 2009, Staermose et al. 2009). In studies using differentiated 3T3-L1 cells, Ald disturbed the expression and secretion of adiponectin and PAI-1 in adipocytes (Li et al. 2011). Consistent with the observations made in adipocytes, incubating preadipocytes with Ald increases the mRNA levels of TNF $\alpha, M C P 1$ (CCL2), and IL6 and decreases the mRNA and protein levels of adiponectin and PPAR $\gamma$ (PPARG). These data suggest that an unfavorable adipokine profile may be created by hyperaldosteronism. However, the molecular and cellular mechanisms underlying the direct regulation of adipokines by Ald remain to be established.

Adipocytes secrete a variety of factors (adipokines), which are hormonally active molecules with widespread effects throughout the body. Apelin has been proposed as a novel beneficial adipokine related to insulin resistance, cardiovascular risk factors, hypertension, and obesity (Boucher et al. 2005, Castan-Laurell et al. 2008, Sonmez et al. 2010). Apelin is the endogenous ligand of the G-protein-coupled receptor (APJ; Tatemoto et al. 1998). Apelin and APJ (APLNR) mRNAs are widely expressed in mammalian tissues and are associated with functional effects in both the CNS and peripheral tissues (Carpene et al. 2007). Apelin and APJ constitute a relatively new peptidic system that has multiple protective functions in cardiovascular homeostasis and metabolism (Castan-Laurell et al. 2011). Given the potential benefits of apelin, elucidating the mechanisms involved in apelin regulation will extend our understanding of the properties of this novel adipokine. Recent studies have demonstrated that insulin exerts a direct control on apelin gene expression in adipocytes, which influences blood concentrations of apelin (Boucher et al. 2005). In addition, besides insulin, GH, and eicosapentaenoic acid are also potent stimulators of apelin expression and secretion in 3T3-L1 adipocytes (Kralisch et al. 2007, Lorente-Cebrian et al. 2010). However, other factors such as $T N F \alpha$, hypoxia, and
PPAR- $\gamma$ coactivator-1a (PGC1a) positively regulate apelin expression in adipose tissue (Daviaud et al. 2006, Glassford et al. 2007, Mazzucotelli et al. 2008, Geiger et al. 2011). Interestingly, there are few known negative modulators of apelin expression in adipocytes; only glucocorticoids have been found to reduce apelin mRNA levels in 3T3-L1 cells (Wei et al. 2005). A lot of evidence points to a direct interaction between the apelin-APJ system and the renin-angiotensin system (RAS) at both molecular and transcriptional levels (Barnes et al. 2010). A recent study has shown that the inhibition of Ang II synthesis or Ang II receptors increases apelin expression and secretion and that Ang II peculiarly regulates apelin secretion and expression in 3T3-L1 adipocytes in a biphasic manner, which reveals a new regulatory mechanism for apelin secretion from adipocytes (Hung et al. 2011, Than et al. 2012). Considering the role of the apelin-APJ system and the RAS in cardiovascular homoeostasis and MS, we hypothesized that there was a counter-regulatory interaction between the apelin-APJ system and Ald.

The purpose of this study was to explore the regulatory effects of Ald on apelin expression and secretion and the underlying mechanisms in 3T3-L1 adipocytes. Our results showed that an acute Ald overloading was inversely related to plasma apelin levels and adipose tissue apelin production and that Ald down-regulated apelin expression in a dose- and time-dependent manner. Furthermore, the intracellular pathways involved in these effects were also studied. The information obtained may provide novel clues about the regulatory role of Ald in adipokine expression in adipose tissue and suggests that apelin is an important target for excess Ald-associated cardiovascular events and metabolic disorders.

\section{Materials and methods}

\section{Reagents and antibodies}

Ald, dexamethasone (Dex), insulin, isobutylmethylxanthine, cycloheximide (CHX), actinomycin D (Act-D), RU486, eplerenone (EP), spironolactone (SP), LY294002, PD98059, SB203580, BMS345541, H89, and SP600125 were purchased from Sigma Chemical Co. TRIzol reagent was purchased from Invitrogen. The real-time PCR (RT-PCR) kit was obtained from Takara (Shiga, Japan). The DIG Northern Starter Kit was purchased from Roche. Antibodies against mouse apelin, glucocorticoid receptor (GR), mineralocorticoid receptor (MR), and $\beta$-actin were ordered from Santa Cruz Biotechnology, while phosphoand total-p38 MAPK antibodies, secondary anti-rabbit and

Published by Bioscientifica Ltd. 
anti-mouse antibodies were purchased from Cell Signaling Technology (Beverly, MA, USA). ECL reagents were purchased from Pierce (Rockford, IL, USA).

\section{Experimental animals}

Male C57BL/6J mice (10 weeks old) were purchased from the Shanghai Laboratory Animal Center at the Chinese Academy of Science (CAS). The mice were maintained in accordance with the NIH Guide for the Care and Use of Laboratory Animals and were fed with normal chow in a standard animal room with $12 \mathrm{~h}$ light: $12 \mathrm{~h}$ darkness cycles. After 2 weeks of adaptive feeding and fasting overnight, the mice (weight: $26.2 \pm 2.1 \mathrm{~g}$ ) were administered one i.p. injection of $1 \mathrm{mg} / \mathrm{kg}$ of Ald or of a vehicle $(150 \mathrm{mM} \mathrm{NaCl}, 5 \%$ ethanol). After the injection, the mice were killed at the following time points: 0,3 , and $6 \mathrm{~h}$ respectively ( $n=8$, each group). To avoid the hemodynamic effects of the mineralocorticoid, the mice were killed after the injection by cervical dislocation under $3 \%$ isoflurane anesthesia. Blood samples were obtained from the mice to measure plasma Ald and apelin concentrations by RIA and ELISA. Visceral adipose tissues were collected and immediately frozen in liquid nitrogen for molecular studies.

\section{Culture and differentiation of 3T3-L1 cells}

The cultures of 3T3-L1 fibroblasts were obtained from American Type Culture Collection (Rockville, MD, USA) and were induced to differentiate into mature adipocytes as described previously (Li et al. 2004). The experiments were conducted on day 8 after induction when more than $90 \%$ of the cells exhibited adipocyte morphology. The 3T3-L1 adipocytes were starved for $12 \mathrm{~h}$ in serum-free DMEM containing $0.1 \%$ BSA prior to treatment. For the in vitro experiments, cells between passages 8 and 10 were used.

\section{RT-PCR and northern blot analysis}

Total RNA was isolated from mature 3T3-L1 adipocytes and mouse epididymal adipose tissue using TRIzol reagent according to the manufacturer's instructions. First-strand cDNA was synthesized from total RNA using the RT System (A3500, Promega). Gene expression was analyzed using quantitative RT-PCR with the $2^{-\Delta \Delta C T}$ relative quantitative method and an ABI Prism 7300 RT-PCR instrument (Applied Biosystems). The primers used in the experiments are given in Supplementary Table 1, see section on supplementary data given at the end of this article. The mRNA levels of all the genes were normalized to that of an internal control, 36B4. Northern blot analysis was performed using the Digoxigenin Northern Starter Kit according to the manufacturer's protocol (Hu et al. 2005). Target fragments of mouse apelin and 36B4 were individually cloned into the pGEM-T Easy vector and were confirmed by sequencing. A DIG-labeled probe was generated by transcription with SP6 or T7 RNA polymerase using the DIG Northern Starter Kit. In the northern blot analysis, $15 \mu \mathrm{g}$ of total RNA was used for each lane. Changes in the apelin mRNA levels were determined by first correcting for the densitometric intensity of 36B4 for each sample.

\section{Hormone receptor knockdown}

The mouse $G r$ (Nr3c1) siRNA (si-GR) (J-045970-10 NR3C1) and nontargeting control siRNA (scr-GR) (\#2 D-00121002-05) were purchased from Dharmacon (Lafayette, CO, USA). Three days after induction, 3T3-L1 adipocytes were transfected with $2 \mu \mathrm{M}$ of siRNA for $24 \mathrm{~h}$ according to the manufacturer's protocol. The transfected cells were cultured in a six-well plate for an additional 2 days and were subsequently starved for $12 \mathrm{~h}$ before the treatment. $\mathrm{Gr}$ knockdown was verified using quantitative RT-PCR and western blot analysis.

\section{Western blot analysis}

Western blot analysis was performed as described previously (Ma et al. 2011). Proteins were separated using a $12 \%$ SDS-PAGE gel followed by electrophoretic transfer to nitrocellulose membranes (Whatman, GE Healthcare). The membranes were blocked for $1 \mathrm{~h}$ and were then incubated overnight at $4{ }^{\circ} \mathrm{C}$ with anti-APLN antibody (1:2000), anti-GR antibody (1:1000), anti-MR antibody (1:500), and anti-p38 MAPK antibody (1:2000). Subsequently, the membranes were incubated for $2 \mathrm{~h}$ at room temperature with a goat anti-mouse or a goat antirabbit IgG HRP-conjugated secondary antibody (1:2000). The signal was detected using the ECL system.

\section{Biochemical assays}

The plasma Ald levels were measured using an RIA Kit (Diagnostic Products Corp., Los Angeles, CA, USA). The apelin levels were quantified using a nonselective apelin ELISA Kit (Phoenix Pharmaceuticals, Belmont, CA, USA) in accordance with the manufacturer's instructions.

Published by Bioscientifica Ltd. 


\section{Statistical analysis}

The data are presented as the mean \pm s.D. All the experiments were independently performed at least three times. Statistical significance was calculated using the two-tailed Student's $t$-test and $P<0.05$ was considered significant.

\section{Results}

\section{Regulation of apelin by Ald in vivo}

To investigate the acute effects of Ald in vivo, we administered a direct i.p. injection of $1 \mathrm{mg} / \mathrm{kg}$ Ald to male C57BL6/J mice at intervals of 0,3 , and $6 \mathrm{~h}$. The dosage was chosen according to the study of Lopez-Andres et al. (2008). The plasma Ald and apelin concentrations of the experimental and control groups were determined by RIA and ELISA (Fig. 1A and B). The serum Ald concentrations peaked $(710 \pm 22.3$ vs $178 \pm 12.5 \mathrm{pg} / \mathrm{ml}$ in the control group; $P<0.01) 3 \mathrm{~h}$ after the injection and then gradually declined (while remaining significantly elevated) to $336 \pm 28.7 \mathrm{pg} / \mathrm{ml}$ at $6 \mathrm{~h}$. By contrast, a remarkable decrease

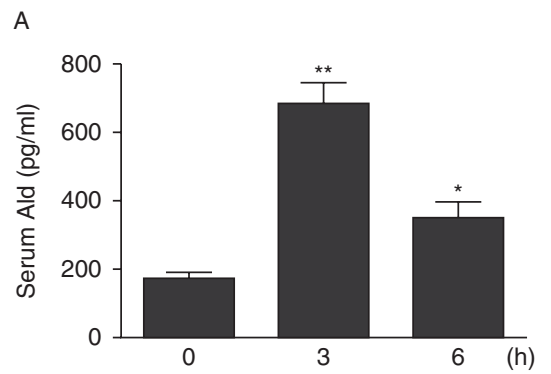

C
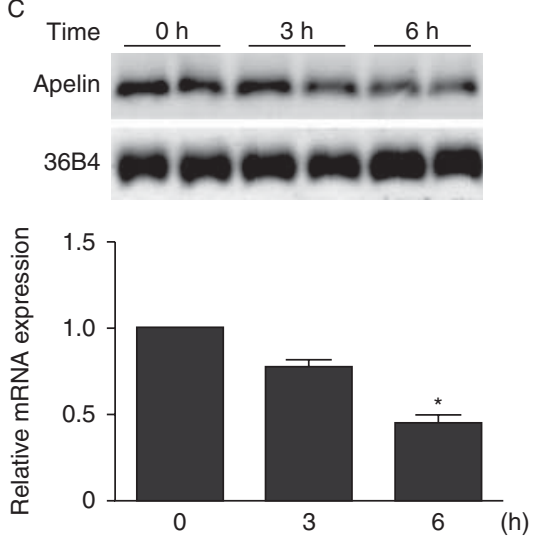

\section{Figure 1}

The effects of Ald on apelin expression and secretion. C57B/6/J mice were administered a single i.p. injection of $1 \mathrm{mg} / \mathrm{kg}$ of Ald (experimental mice) or of a vehicle alone (control mice) and were killed at the indicated time points. ( $A$ and $B$ ) The plasma concentrations of Ald and apelin were measured with ELISA. (C and D) The apelin mRNA and protein levels in visceral adipose tissue were measured using northern and western blot in the plasma levels of apelin $(290 \pm 27.2$ vs $610 \pm 20.3 \mathrm{pg} / \mathrm{ml}$ in the control group; $P<0.05)$ was observed $6 \mathrm{~h}$ after the injection. Additionally, we detected apelin mRNA expression in the visceral adipose tissues of the mice. Ald caused a nearly 55\% reduction in the apelin mRNA levels by $6 \mathrm{~h}$ after the treatment, as demonstrated by the northern blot analysis results $(P<0.05$; Fig. $1 C)$. Consistent with the apelin mRNA levels, the apelin protein levels were also markedly reduced at $6 \mathrm{~h}(P<0.05$; Fig. 1D). Our results demonstrate that a severe excess of Ald significantly decreases the plasma apelin levels and apelin expression in adipose tissue. However, whether apelin production in adipose tissue influences the blood concentrations of apelin after Ald treatment is an interesting issue that requires further study.

\section{Ald reduced apelin expression in a concentration- and time-dependent manner}

The low levels of apelin in response to hyperaldosteronism could be the result of a direct effect of Ald or that of

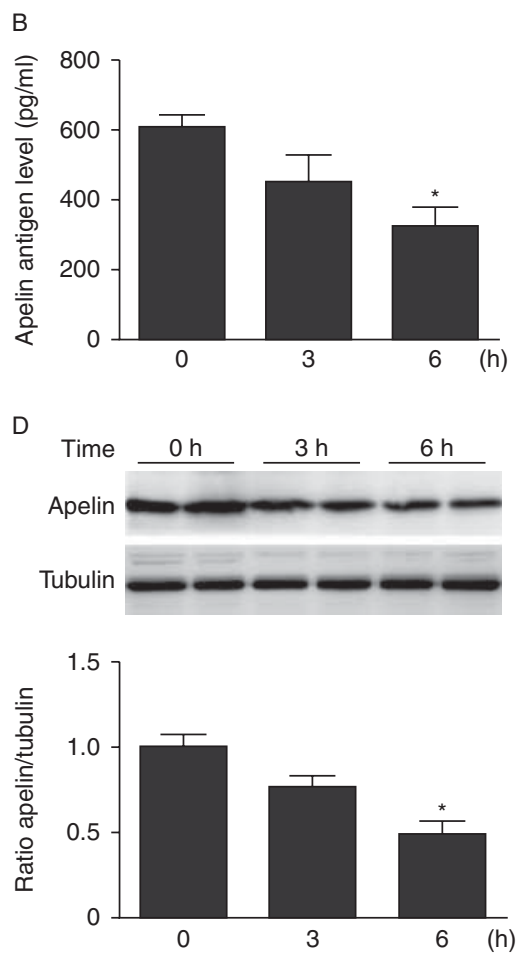

analyses. The relative mRNA levels were normalized to that of 36B4 mRNA and the protein levels were normalized to that of tubulin. The bands were quantified by densitometry using the Quantity One system. The results represent the mean \pm S.D. of each group of mice $(n=8)$. ${ }^{*} P<0.05$ and $* * P<0.01$ vs the control group $(0 \mathrm{~h})$. http://jme.endocrinology-journals.org DOI: 10.1530/JME-13-0025
(C) 2013 Society for Endocrinology Printed in Great Britain 
the mechanisms originating from an excess of Ald. To evaluate the direct effects of Ald on apelin expression and secretion, we performed dose- and time-dependent experiments in 3T3-L1 adipocytes. The incubation of 3T3-L1 adipocytes with different concentrations of Ald $(10 \mathrm{nM}-10 \mu \mathrm{M})$ for $24 \mathrm{~h}$ decreased the apelin mRNA levels in a dose-dependent manner $(P<0.05$ and $P<0.01$ respectively; Fig. 2A). It is worth noting that Ald significantly down-regulated apelin expression only at pathological concentrations ( 1 and $10 \mu \mathrm{M})$. When 3T3-L1 adipocytes were exposed to $10 \mu \mathrm{M}$ Ald, an early reduction in apelin expression occurred at $3 \mathrm{~h}$ and a maximal decrease of $85 \%$ was detected at $24 \mathrm{~h}(P<0.01$; Fig. $2 \mathrm{~B})$. The decrease in apelin protein expression paralleled the change in the mRNA levels, whereas the decline in apelin antigen secretion lagged behind the mRNA decrease (Fig. 2C and D). Our results demonstrate that

A
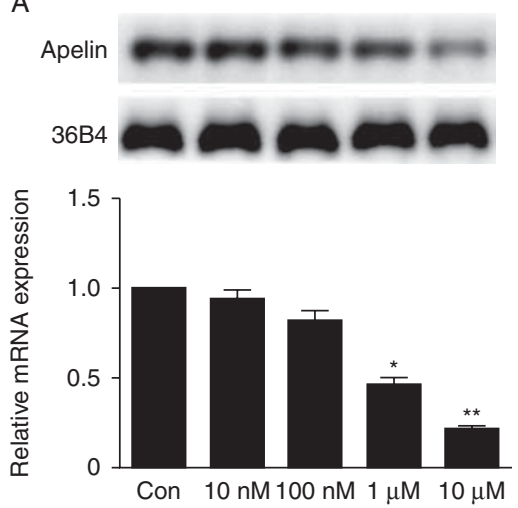

C

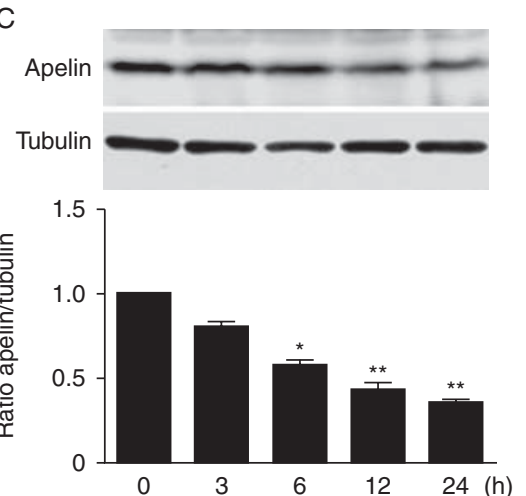

Figure 2

Ald regulates apelin expression and secretion in a dose- and timedependent manner. After serum deprivation for $12 \mathrm{~h}$, 3T3-L1 adipocytes were incubated with the indicated concentrations of Ald for time periods of different lengths. ( $A$ and $B$ ) Northern blot analysis revealed a dose (24 h)and time $(10 \mu \mathrm{M})$-dependent down-regulation of apelin mRNA expression.
Ald affects apelin expression and secretion in a time- and concentration-dependent manner.

To determine whether the Ald-induced decrease in the apelin mRNA levels was a direct effect, we first studied the disappearance of apelin mRNA after treatment with Act-D $(5 \mu \mathrm{g} / \mathrm{ml})$ for $1,2,4,8$, or $12 \mathrm{~h}$. As shown in Fig. 3A, the kinetics of mRNA disappearance were similar between the control and Ald-treated cells (the half-life was $4 \mathrm{~h} 45 \mathrm{~min}$ and $5 \mathrm{~h} 5 \mathrm{~min}$ for the control and Ald treatments respectively), indicating that Ald decreased apelin gene transcription rather than increasing mRNA degradation. The half-life of apelin in 3T3-L1 cells was consistent with prior reports made for 3T3-F442A cells (Boucher et al. 2005). Next, 3T3-L1 adipocytes were pretreated with the protein synthesis inhibitor CHX $(2 \mu \mathrm{g} / \mathrm{ml})$ for $30 \mathrm{~min}$, and then Ald $(10 \mu \mathrm{M})$ was added for $12 \mathrm{~h}$. The northern blot analysis results indicated that

B
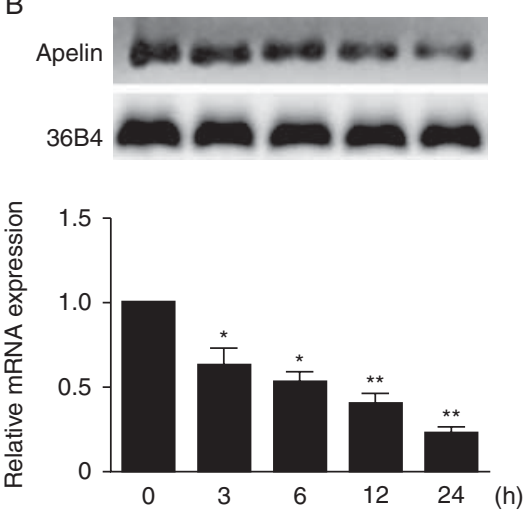

D

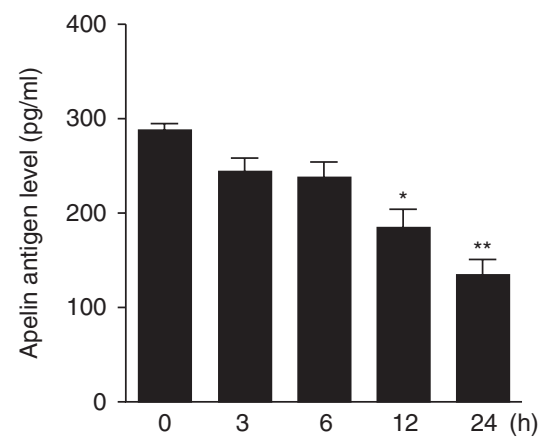

(C and D) Time-dependent $(10 \mu \mathrm{M})$ decreases in apelin protein production and antigen secretion were revealed by the western blot analysis and ELISA respectively. The values represent the mean \pm s.D. of three independent experiments. $* P<0.05$ and $* * P<0.01$ vs the control (Con).

Published by Bioscientifica Ltd. 


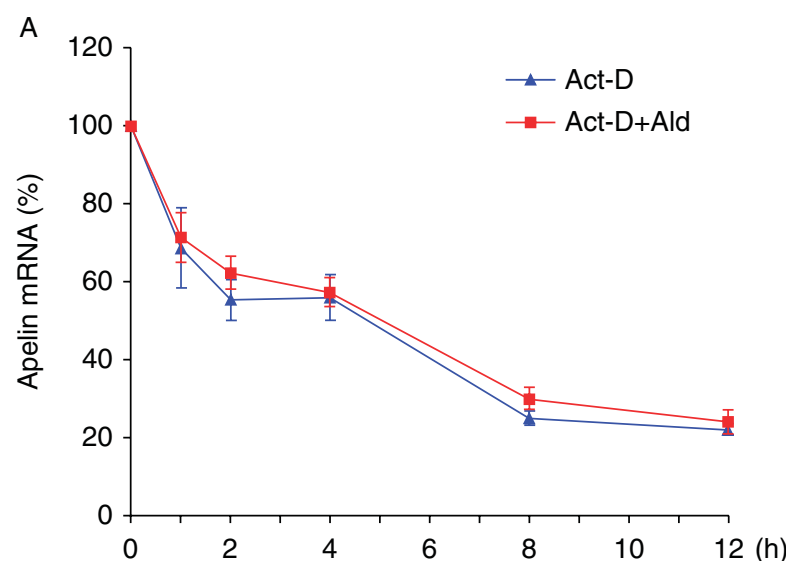

B

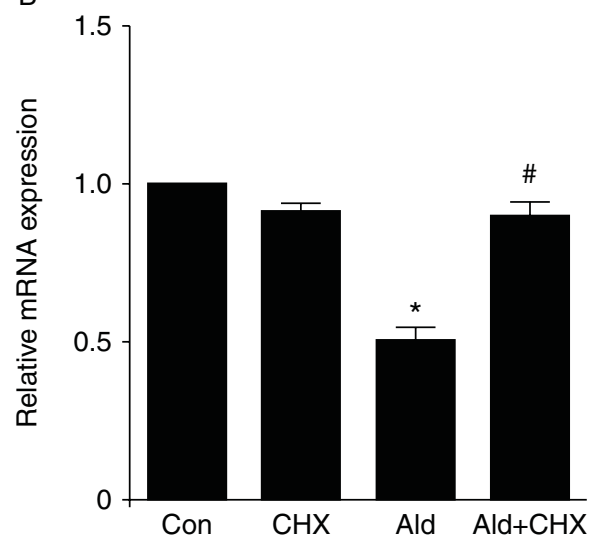

Figure 3

Act-D and CHX on the effects of Ald. (A) 3T3-L1 adipocytes were preincubated with Act-D $(5 \mu \mathrm{g} / \mathrm{ml})$ for $30 \mathrm{~min}$ and then treated (or not) with Ald $(1 \mu \mathrm{M})$ for $1,2,4,8$, or $12 \mathrm{~h}$. (B) 3T3-L1 adipocytes were preincubated with $\mathrm{CHX}(2 \mu \mathrm{g} / \mathrm{ml})$ for $30 \mathrm{~min}$ and then treated (or not) with Ald $(1 \mu \mathrm{M})$ for $12 \mathrm{~h}$. The apelin mRNA levels were measured using a northern blot analysis and are expressed as a percentage of the control. The data represent the mean \pm s.D. of three separate experiments. ${ }^{*} P<0.05$ vs control (Con) and ${ }^{\#} P<0.05$ vs Ald.

CHX blunted the apelin mRNA decrease $(P<0.01$; Fig. 3B), suggesting that de novo protein synthesis was involved in apelin transcription. These findings indicate that the regulation of apelin by Ald is mediated through a new genomic effect in adipocytes.

\section{Mechanisms of mineralocorticoid and glucocorticoid on apelin expression in adipocytes}

Recent data have indicated that Dex can drastically reduce apelin mRNA levels within the physiological concentration range in 3T3-L1 adipocytes (Wei et al. 2005); however, the underlying mechanism remains unclear. Therefore, we measured apelin mRNA expression, protein production, and secretion in 3T3-L1 adipocytes treated with $100 \mathrm{nM}$ of Dex and/or $10 \mu \mathrm{M}$ of Ald. As shown in Fig. 4A, when administered alone, both Dex and Ald significantly decreased the mRNA expression of apelin by similar proportions (Dex 74\% vs Ald 78\%). However, cotreatment with Dex and Ald produced no additive effect on apelin expression (Fig. 4A). Parallel to the mRNA levels, apelin protein production in the adipocytes was also reduced significantly after the treatment (Dex 55\% vs Ald 58\% vs cotreatment 61\%; Fig. 4B). Moreover, the protein secretion analysis by ELISA indicated that there was a significant reduction in the secretion of apelin into the medium $(280+12.3 \mathrm{pg} / \mathrm{ml}$ in the control cells vs $145+11.8 \mathrm{pg} / \mathrm{ml}$ in the Dex-treated cells vs $132.5+15.8 \mathrm{pg} / \mathrm{ml}$ in the Ald-treated cells vs $127.5+16.2 \mathrm{pg} / \mathrm{ml}$ in the cotreated cells; Fig. 4C). Apelin and APJ are often colocalized in the same tissues and display similar variations of expression. In additional experiments, we also detected Apj mRNA expression by RT-PCR after the treatment; there were no significant differences among the treatments (Fig. 4D).

As Ald binds to the GR and MR with a different affinity, low hormone concentrations will bind to the MR and high hormone concentrations will bind to the GR. We first confirmed the expression of both receptors in the adipocytes. Western blot analysis and RT-PCR revealed that the GR is much more abundant than the MR in the adipocytes (Fig. 5A). To investigate whether the MR or GR was involved in the Ald-induced apelin decrease, 3T3-L1 adipocytes were pretreated with the specific receptor antagonists for $30 \mathrm{~min}$, then Ald $(1$ or $10 \mu \mathrm{M})$, Dex $(100 \mathrm{nM})$ or both were added and finally the cells were incubated for $24 \mathrm{~h}$. The northern blot analysis and ELISA results revealed that the effect of Ald was not reversed by the competitive MR antagonist (SP) or by the noncompetitive MR antagonist (EP); however, it was suppressed by the selective GR antagonist (RU486) at both low and high Ald concentrations $(P<0.01$; Fig. $5 \mathrm{~B}, \mathrm{C}$ and $\mathrm{D})$. Furthermore, RU486 could also reverse the effect of the glucocorticoid as well as the effect of its combination with Ald (Fig. 5C and D). These results suggest that the effects of both Ald and Dex on apelin expression were mediated via the GR but not via the MR in 3T3-L1 adipocytes.

To confirm the results of the antagonist experiments, we examined whether a specific reduction in GR expression could modulate apelin expression in the adipocytes. Using GR-specific siRNA, we induced marked decreases in the GR mRNA (78\%) and protein (80\%) expression compared with the effects of scrambled siRNA (scr-GR; Fig. 5E). Subsequently, 3T3-L1 cells were treated

Published by Bioscientifica Ltd. 
A
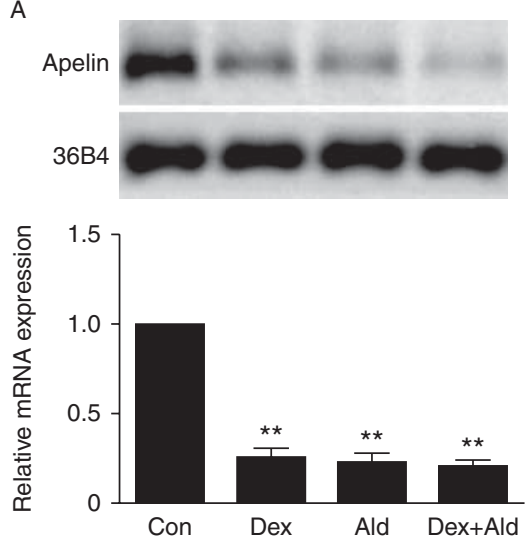

C

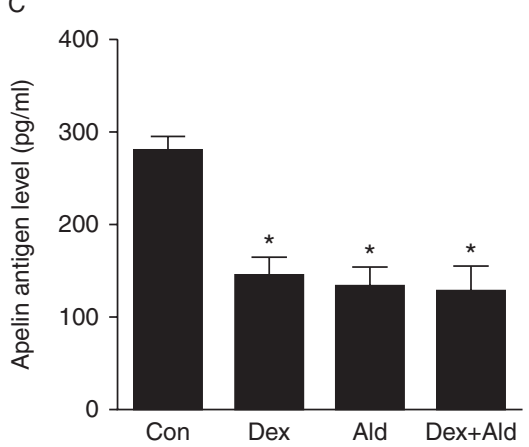

B

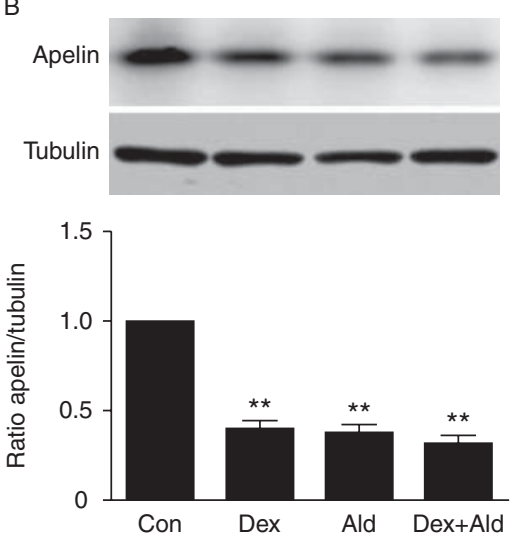

D

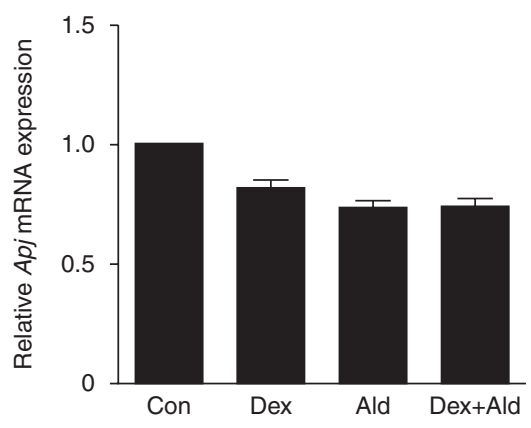

\section{Figure 4}

Comparison of the effects of Dex and Ald in 3T3-L1 adipocytes. After serum deprivation for $12 \mathrm{~h}, 3 \mathrm{T3}-\mathrm{L} 1$ adipocytes were incubated with Ald $(10 \mu \mathrm{M})$, Dex $(100 \mathrm{nM})$, or Ald + Dex for $24 \mathrm{~h}$. (A and B) The apelin mRNA expression and protein production in adipocytes were analyzed using northern and western blot analyses. (C) The levels of apelin antigen in a conditioned

with Ald $(10 \mu \mathrm{M})$ or Dex $(100 \mathrm{nM})$ for $24 \mathrm{~h}$, and apelin mRNA expression and protein secretion were measured. As shown in Fig. 5F, neither treatment significantly depressed apelin expression. Our findings demonstrate that both mineralocorticoids and glucocorticoids could activate the GR to reduce apelin expression, suggesting that GR response elements (GREs) may be present in the apelin promoter. Fortunately, we identified five candidate GREs in the $5^{\prime}$ flanking region $(-2.5 \mathrm{~kb})$ of the murine apelin gene using the program AliBaba 2.1 (Supplementary Figure 1, see section on supplementary data given at the end of this article).

\section{The p38 MAPK signaling pathway is involved in the effect of Ald}

A variety of signaling molecules and intracellular signaling cascades affected by Ald have been identified (Boldyreff \& Wehling 2003). To investigate the possible medium were measured with ELISA. (D) The Apj mRNA expression in 3T3-L1 adipocytes was analyzed with RT-PCR, and the expression in the control cells was normalized to 1.0. The data represent the mean \pm s.D. of three independent experiments. ${ }^{*} P<0.05$ and ${ }^{* *} P<0.01$ vs the control (Con).

pathway of Ald-mediated effects on apelin expression, we used specific chemical inhibitors to investigate the potential participation of other intracellular cascades activated by Ald in 3T3-L1 adipocytes. After a 30-min pretreatment with the p38 MAPK inhibitor SB203580 $(20 \mu \mathrm{M})$, the p42/44 MAPK inhibitor PD98059 $(25 \mu \mathrm{M})$, the JNK inhibitor SP600125 $(20 \mu \mathrm{M})$, the PI3K inhibitor LY294002 $(10 \mu \mathrm{M})$, the IKK inhibitor BMS $(10 \mu \mathrm{M})$, or the chemical antioxidant $N$-acetylcysteine $(1 \mu \mathrm{M}), 3$ T3-L1 adipocytes were incubated with Ald $(10 \mu \mathrm{M})$ for $24 \mathrm{~h}$. As shown in Fig. 6A, only SB203580 modulated the effect of Ald, by partially reversing it $(P<0.05)$. However, SB203580 completely reversed the suppression of apelin expression at the 6 -h time point $(P<0.01$; Fig. 6B). In addition, SB203580 could not block the effect of Dex (Fig. 6B). None of the above-mentioned chemicals could affect apelin expression in the absence of Ald (data not shown). Next, a time-response analysis of p38 MAPK activation induced by Ald revealed a significant 2.8-fold activation of p38

Published by Bioscientifica Ltd. 
A
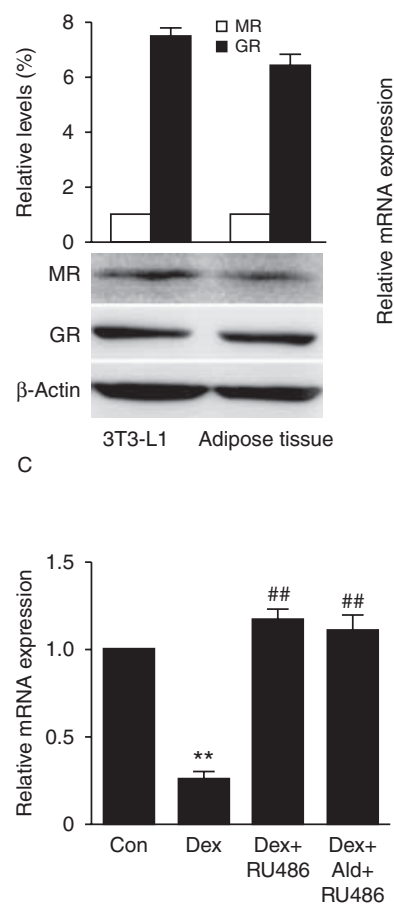

E

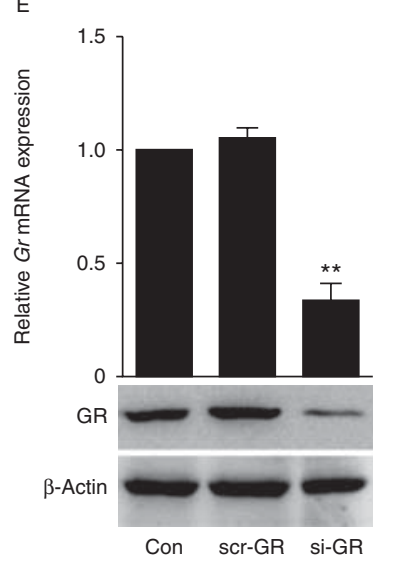

B
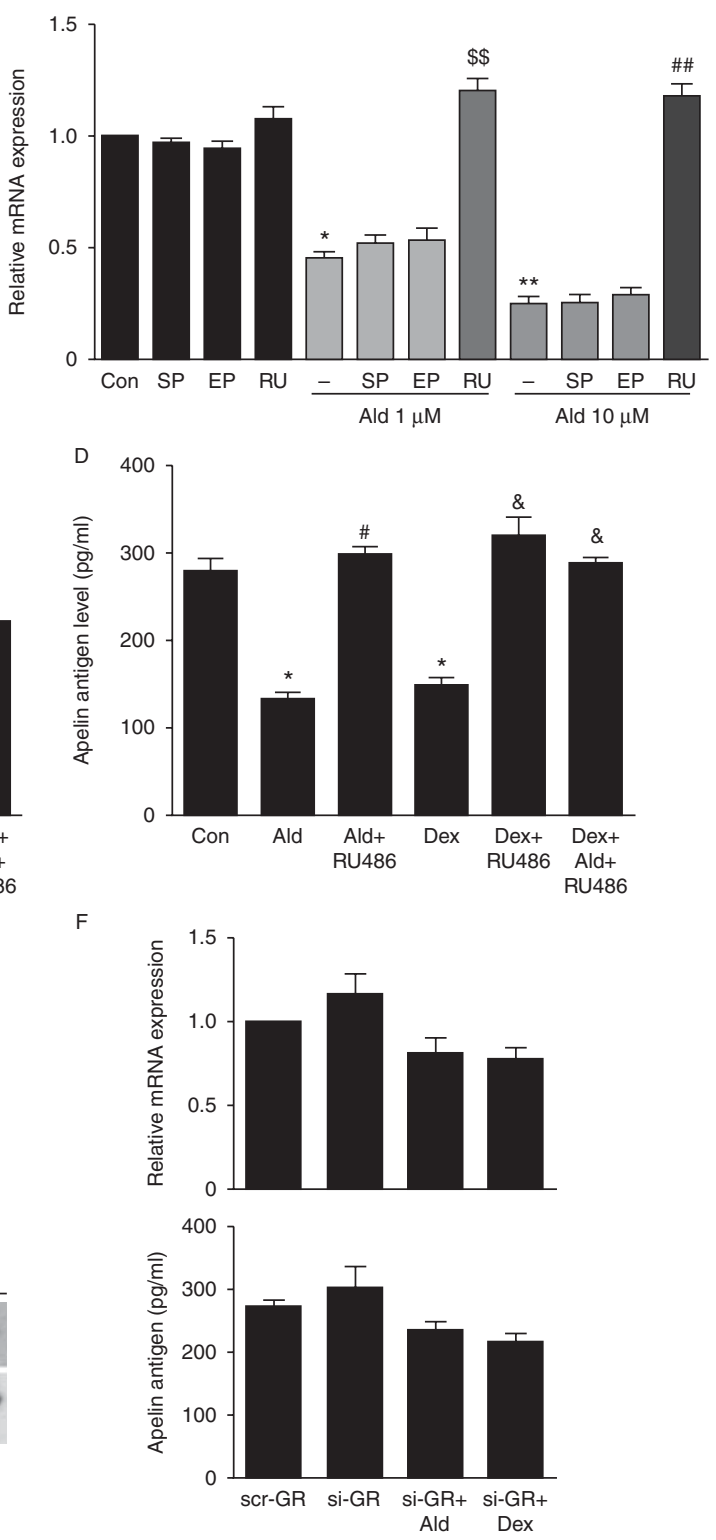

\section{Figure 5}

GR and MR involved in the effects of mineralocorticoids and glucocorticoids. (A) RT-PCR and western blot analysis were used to examine the GR and MR expression in 3T3-L1 adipocytes and in mouse epididymal adipose tissue. The mRNA levels were normalized to that of 36B4, and the GR levels are expressed relative to the MR levels. (B) 3T3-L1 adipocytes were pretreated with $10 \mu \mathrm{M} \mathrm{SP}, 10 \mu \mathrm{M}$ EP or $1 \mu \mathrm{M}$ RU486 (RU) for $30 \mathrm{~min}$ and were then incubated with 1 or $10 \mu \mathrm{M}$ of Ald for $24 \mathrm{~h}$. (C and D) 3T3-L1 adipocytes were pretreated with $1 \mu \mathrm{M}$ RU and were then subjected to $100 \mathrm{nM}$ Dex, $10 \mu \mathrm{M}$ Ald, or both for $24 \mathrm{~h}$. The apelin mRNA levels were detected by a northern blot analysis and expressed as quantified bands. Apelin antigen secretion was analyzed by ELISA. (E) RT-PCR and western blot analysis were used to examine the GR expression in Gr knockdown adipocytes. (F) In Gr knockdown adipocytes treated with Ald (10 $\mu \mathrm{M})$ and Dex (100 nM) for $24 \mathrm{~h}$, apelin mRNA expression and antigen secretion were measured by northern blot analysis and ELISA. The results represent the mean \pm s.D. of three separate experiments, $* P<0.05$ and $* * P<0.01$ vs the control. ${ }^{\$} P<0.01$ vs Ald $(1 \mu \mathrm{M})$. ${ }^{\#} P<0.05$ and ${ }^{\# \#} P<0.01$ vs Ald $(10 \mu \mathrm{M})$. ${ }^{\&} P<0.05$ vs Dex (100 nM); Con, control.
MAPK phosphorylation 30 min after the addition of Ald $(P<0.01)$; however, this phosphorylation gradually diminished to the baseline level by $6 \mathrm{~h}$ (Fig. 6C). Additionally, Ald-induced p38 MAPK phosphorylation was partly attenuated by the addition of RU486 and SB203580 at 30 min $(P<0.05$; Fig. 6D). These findings indicate that Ald-induced p38 MAPK activation in adipocytes partially involves a GR-dependent pathway. 
A

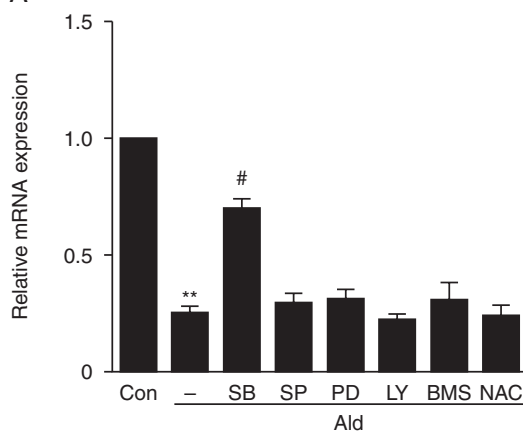

C
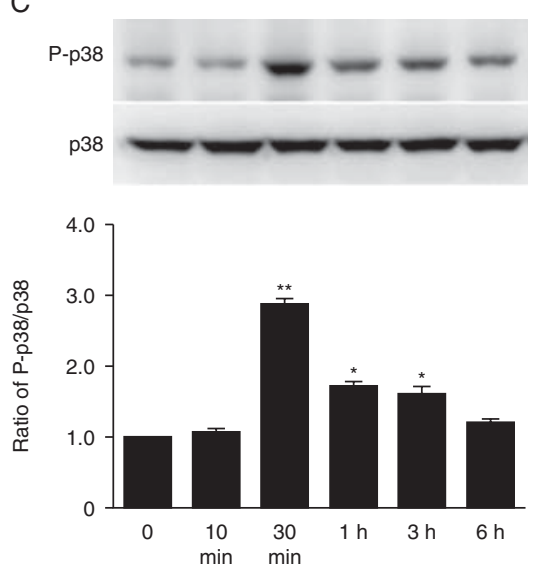

B

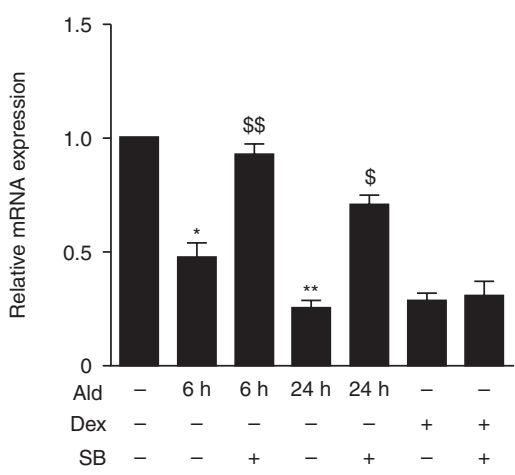

$\mathrm{D}$
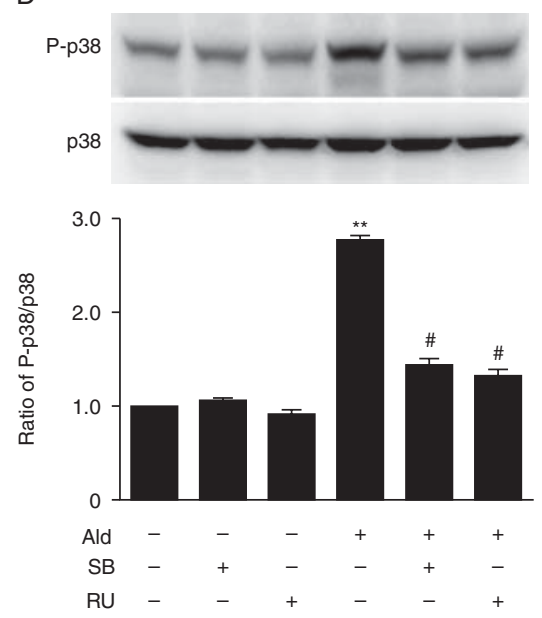

\section{Figure 6}

Ald-induced apelin down-regulation mediated via p38 MAPK. (A) 3T3-L1 adipocytes were incubated with $10 \mu \mathrm{M}$ Ald for $24 \mathrm{~h}$ in the absence or presence of the p38 MAPK inhibitor SB203580 (SB, $20 \mu \mathrm{M})$, the p42/44 MAPK inhibitor PD98059 (PD, $25 \mu \mathrm{M})$, the JNK inhibitor SP600125 (SP, $20 \mu \mathrm{M})$, the PI3K inhibitor LY294002 (LY, $10 \mu \mathrm{M})$, the IKK inhibitor BMS $(10 \mu \mathrm{M})$, or the chemical antioxidant $N$-acetylcysteine (NAC, $1 \mu \mathrm{M}$ ). (B) 3T3-L1 adipocytes were pretreated with SB $(20 \mu \mathrm{M})$ for 30 min and then incubated with Ald $(10 \mu \mathrm{M})$ for 6 or $24 \mathrm{~h}$ or incubated with Dex $(100 \mathrm{nM})$ for $24 \mathrm{~h}$. The apelin mRNA levels were measured by a northern blot analysis and are expressed as the percentages of the levels of the control. (C) 3T3-L1 adipocytes were incubated with Ald $(10 \mu \mathrm{M})$, and the level of p38 MAPK phosphorylation was examined by a western blot analysis at the indicated time points. (D) 3T3-L1 adipocytes were pretreated with SB $(10 \mu \mathrm{M})$ or RU $(1 \mu \mathrm{M})$ for $30 \mathrm{~min}$, and then Ald $(10 \mu \mathrm{M})$ was added for $30 \mathrm{~min}$. The level of p38 MAPK phosphorylation was measured by a western blot analysis. The ratios of phosphorylated to nonphosphorylated p38 MAPK protein recorded at the indicated points are shown in the figure. The results represent the mean \pm s.D. of three separate experiments. ${ }^{*} P<0.05$ and ${ }^{* *} P<0.01$ vs the control (Con); ${ }^{*} P<0.05$ vs Ald $(10 \mu \mathrm{M}) ;{ }^{\$} P<0.05$ vs Ald $(6 \mathrm{~h}) ;{ }^{\$} P<0.01$ vs Ald $(24 \mathrm{~h})$.

\section{Discussion}

This study reports some completely novel findings about the relationship between Ald and apelin: i) elevated Ald levels are inversely related to plasma apelin levels and adipose tissue apelin production. ii) In vitro, Ald downregulates apelin expression and secretion in a dose- and time-dependent manner. Both mineralocorticoids and glucocorticoids significantly down-regulate apelin expression and secretion through GR activation, although they produce no synergistic effect. iii) The activation of the p38 MAPK signaling pathway is partially responsible for

the regulation of apelin expression by Ald and may be downstream of GR activation, suggesting a nongenomic interaction between the p38 MAPK pathway and the genomic effects of Ald.

The relationship between Ald and obesity is of major clinical importance, because both play important roles in the pathogenesis of the MS, cardiovascular disease, and resistant hypertension. In obesity, the factors (adipokines) released from fat cells directly or indirectly stimulate Ald secretion from the adrenal cortex, which have to be considered as those involved in an additional mechanism

Published by Bioscientifica Ltd. 
of excess Ald production. However, the crosstalk between Ald and adipokines is even more limited and is an area of growing interest. According to a study by Iacobellis et al. (2010), patients with PA have significantly higher leptin levels, lower adiponectin levels, elevated circulating resistin and a higher rate of the MS compared with individuals with essential hypertension. Similar to adiponectin, apelin is considered to be a beneficial adipokine, one that exerts its useful effects to prevent obesity-associated diseases and to promote cardiovascular homeostasis (Barnes et al. 2010). Recent studies have shown that both systemic and local AngII can reduce apelin expression in adipocytes and that a blockade of the RAS ameliorates apelin expression and secretion in 3T3-L1 adipocytes, suggesting a counter-regulatory interaction between apelin and the RAS (Hung et al. 2011, Than et al. 2012). Thus, on the basis of our understanding of the RAS, our study provides a novel example of a direct effect of Ald on apelin expression in adipocytes and supports the existence of crosstalk between the renin-angiotensinaldosterone system and the vasoactive peptide apelin, which may play an important role in cardiovascular and metabolic homeostasis.

Previous studies have reported that Dex reduced apelin mRNA levels in 3T3-L1 adipocytes in a dosedependent manner (Boucher et al. 2005). In our study, we thoroughly examined the direct effects of both glucocorticoids and mineralocorticoids on apelin expression in adipocytes. Both corticosteroids significantly down-regulated apelin expression, but no additive effect was observed. Studies on GR antagonists and GR knockdown have suggested that both Dex and Ald share the same receptor (GR) to regulate the transcription of apelin in adipocytes. We speculated that both corticosteroids might share a same pathway in regulating apelin expression in adipocytes. Additional evidence would support our hypothesis: i) GRs are more abundant than MRs in adipocytes (Fig. 5A). ii) Several GREs are located in the promoter of the apelin gene (Supplementary Figure 1). iii) According to a previous report, nanomolar concentrations of Ald activate its cognate receptor, whereas 100-fold higher concentrations of Ald are necessary to activate GRs (Hellal-Levy et al. 1999). Consistent with our reports, Ald has been shown to decrease adiponectin levels and increase PAI-1 expression via GRs, with the maximal effect being achieved with $10 \mu \mathrm{M}$ of Ald in 3T3-L1 adipocytes (Li et al. 2011). In summary, we provide evidence to understand the overlapping effects between glucocorticoids and Ald on the regulation of the same target gene in adipocytes, thus enhancing our understanding of the crosstalk between the adrenal gland and adipose tissue. The data also suggest that reduced apelin levels may mediate the detrimental effect of excessive Ald and cortisol secretion, which has been implicated in the pathogenesis of hypertension and MS.

The molecular mechanisms of the effects of Ald are complex. The classic genomic effects of Ald are characterized by a latent onset and sensitivity to mRNA and protein synthesis inhibitors (Grossmann et al. 2005). In our study, Ald-induced reduced expression of apelin demonstrated a time-dependent mechanism and the steroid hormone acted mainly through transcriptional effects, and also the effects were sensitive to CHX. All the results presented herein clearly demonstrate that the Ald response is critically dependent on the genomic action of Ald in 3T3-L1 adipocytes. Interestingly, the effect of Ald on apelin expression was partly abolished by SB203580, indicating an effect mediated via p38 MAPK, which always exerts rapid nongenomic effects in an Ald-induced signaling pathway (Callera et al. 2005, Lopez-Andres et al. 2008). Nongenomic effects play a substantial role in the mechanisms by which Ald contributes to the pathogenesis of the MS and cardiovascular disease (Wehling et al. 1992, Funder 2004, Sowers et al. 2009). In addition, Ald-activated p38 MAPK phosphorylation was inhibited to a similar extent by both RU486 and SB203580. On the basis of these results, we speculate that the rapid p38 MAPK phosphorylation elicited by Ald is a nongenomic effect that may alter the genomic effects through an interaction with the GR or phosphorylation of transcriptional cofactors. Our data present a novel example of a crosstalk between nongenomic and genomic signals that may clarify the mechanisms by which Ald induces adipokine dysregulation. Further investigation is needed to clarify this issue.

Our study for the first time produces evidence that Ald is a negative regulator of apelin production in adipocytes, which strengthens the crosstalk between adipose tissue and the adrenal cortex. These findings provide novel insights into the effects of glucocorticoid and mineralocorticoid hormones in the modulation of adipose tissue physiopathology. Considering the beneficial roles of apelin in the cardiovascular system and obesity-associated disorders, we hypothesize that the lower plasma apelin levels found in patients with hypertension, cardiovascular dysfunction or insulin resistance may be caused, to some extent, by elevated circulating Ald levels. Further studies on this issue are needed. Thus, therapeutic manipulation of the apelin-APJ system represents a novel and potential

Published by Bioscientifica Ltd. 
therapeutic mechanism especially for high Ald levels or PA-associated cardiometabolic disorders.

\section{Supplementary data}

This is linked to the online version of the paper at http://dx.doi.org/10.1530/ JME-13-0025.

\section{Declaration of interest}

The authors declare that there is no conflict of interest that could be perceived as prejudicing the impartiality of the research reported.

\section{Funding}

This work was supported in part by the National Natural Science Foundation of China (81200643, 8110553, 31171127, and 81201915) and the outstanding doctor cultivation fund of Shanghai Jiaotong University (YBPY2009007).

\section{Author contribution statement}

All authors contributed to the study conception and design. H J, X-PY, Z-YY, and $\mathrm{M} \mathrm{Z}$ conducted the study. All authors analyzed the data. $\mathrm{H} \mathrm{J}$ and $\mathrm{S}-\mathrm{X} \mathrm{Z}$ wrote the paper.

\section{Acknowledgements}

The authors thank Drs Qinyun Ma and Jie Qiao for advice and assistance.

\section{References}

Barnes G, Japp AG \& Newby DE 2010 Translational promise of the apelinAPJ system. Heart 96 1011-1016. (doi:10.1136/hrt.2009.191122)

Boldyreff B \& Wehling M 2003 Rapid aldosterone actions: from the membrane to signaling cascades to gene transcription and physiological effects. Journal of Steroid Biochemistry and Molecular Biology $\mathbf{8 5}$ 375-381. (doi:10.1016/S0960-0760(03)00202-4)

Boucher J, Masri B, Daviaud D, Gesta S, Guigne C, Mazzucotelli A, Castan-Laurell I, Tack I, Knibiehler B, Carpene C et al. 2005 Apelin, a newly identified adipokine up-regulated by insulin and obesity. Endocrinology 146 1764-1771. (doi:10.1210/en.2004-1427)

Callera GE, Touyz RM, Tostes RC, Yogi A, He Y, Malkinson S \& Schiffrin EL 2005 Aldosterone activates vascular p38MAP kinase and NADPH oxidase via c-Src. Hypertension 45 773-779. (doi:10.1161/01.HYP. 0000154365.30593.d3)

Carpene C, Dray C, Attane C, Valet P, Portillo MP, Churruca I, Milagro FI \& Castan-Laurell I 2007 Expanding role for the apelin/APJ system in physiopathology. Journal of Physiology and Biochemistry 63 359-373. (doi:10.1007/BF03165767)

Carvajal CA, Herrada AA, Castillo CR, Contreras FJ, Stehr CB, Mosso LM, Kalergis AM \& Fardella CE 2009 Primary aldosteronism can alter peripheral levels of transforming growth factor $\beta$ and tumor necrosis factor $\alpha$. Journal of Endocrinological Investigation 32 759-765. (doi:10.3275/6429)

Castan-Laurell I, Vitkova M, Daviaud D, Dray C, Kovacikova M, Kovacova Z, Hejnova J, Stich V \& Valet P 2008 Effect of hypocaloric diet-induced weight loss in obese women on plasma apelin and adipose tissue expression of apelin and APJ. European Journal of Endocrinology 158 905-910. (doi:10.1530/EJE-08-0039)

Castan-Laurell I, Dray C, Attane C, Duparc T, Knauf C \& Valet P 2011 Apelin diabetes, and obesity. Endocrine 40 1-9. (doi:10.1007/ s12020-011-9507-9)

Daviaud D, Boucher J, Gesta S, Dray C, Guigne C, Quilliot D, Ayav A, Ziegler O, Carpene C, Saulnier-Blache JS et al. 2006 TNF $\alpha$ up-regulates apelin expression in human and mouse adipose tissue. FASEB Journal 20 1528-1530. (doi:10.1096/fj.05-5243fje)

Ehrhart-Bornstein M, Arakelyan K, Krug AW, Scherbaum WA \& Bornstein SR 2004 Fat cells may be the obesity-hypertension link: human adipogenic factors stimulate aldosterone secretion from adrenocortical cells. Endocrine Research 30 865-870. (doi:10.1081/ ERC-200044122)

Fallo F, Veglio F, Bertello C, Sonino N, Della Mea P, Ermani M, Rabbia F, Federspil G \& Mulatero P 2006 Prevalence and characteristics of the metabolic syndrome in primary aldosteronism. Journal of Clinical Endocrinology and Metabolism 91 454-459. (doi:10.1210/jc.2005-1733)

Fallo F, Della Mea P, Sonino N, Bertello C, Ermani M, Vettor R, Veglio F \& Mulatero P 2007 Adiponectin and insulin sensitivity in primary aldosteronism. American Journal of Hypertension 20 855-861. (doi:10.1016/j.amjhyper.2007.03.012)

Funder JW 2004 Aldosterone, mineralocorticoid receptors and vascular inflammation. Molecular and Cellular Endocrinology 217 263-269. (doi:10.1016/j.mce.2003.10.054)

Geiger K, Muendlein A, Stark N, Saely CH, Wabitsch M, Fraunberger P \& Drexel H 2011 Hypoxia induces apelin expression in human adipocytes. Hormone and Metabolic Research 43 380-385. (doi:10.1055/ s-0031-1273767)

Glassford AJ, Yue P, Sheikh AY, Chun HJ, Zarafshar S, Chan DA, Reaven GM, Quertermous T \& Tsao PS 2007 HIF-1 regulates hypoxia- and insulininduced expression of apelin in adipocytes. American Journal of Physiology. Endocrinology and Metabolism 293 E1590-E1596. (doi:10.1152/ajpendo.00490.2007)

Grossmann C, Benesic A, Krug AW, Freudinger R, Mildenberger S, Gassner B \& Gekle M 2005 Human mineralocorticoid receptor expression renders cells responsive for nongenotropic aldosterone actions. Molecular Endocrinology 19 1697-1710. (doi:10.1210/ me.2004-0469)

Hellal-Levy C, Couette B, Fagart J, Souque A, Gomez-Sanchez C \& Rafestin-Oblin M 1999 Specific hydroxylations determine selective corticosteroid recognition by human glucocorticoid and mineralocorticoid receptors. FEBS Letters 464 9-13. (doi:10.1016/S0014-5793 (99)01667-1)

Hu SM, Li F, Yu HM, Li RY, Ma QY, Ye TJ, Lu ZY, Chen JL \& Song HD 2005 The mimecan gene expressed in human pituitary and regulated by pituitary transcription factor-1 as a marker for diagnosing pituitary tumors. Journal of Clinical Endocrinology and Metabolism 90 6657-6664. (doi:10.1210/jc.2005-0322)

Hung WW, Hsieh TJ, Lin T, Chou PC, Hsiao PJ, Lin KD \& Shin SJ 2011 Blockade of the renin-angiotensin system ameliorates apelin production in 3T3-L1 adipocytes. Cardiovascular Drugs and Therapy 25 3-12. (doi:10.1007/s10557-010-6274-4)

Iacobellis G, Petramala L, Cotesta D, Pergolini M, Zinnamosca L, Cianci R, De Toma G, Sciomer S \& Letizia C 2010 Adipokines and cardiometabolic profile in primary hyperaldosteronism. Journal of Clinical Endocrinology and Metabolism 95 2391-2398. (doi:10.1210/ jc.2009-2204)

Irita J, Okura T, Manabe S, Kurata M, Miyoshi K, Watanabe S, Fukuoka T \& Higaki J 2006 Plasma osteopontin levels are higher in patients with primary aldosteronism than in patients with essential hypertension. American Journal of Hypertension 19 293-297. (doi:10.1016/j.amjhyper. 2005.08.019)

Kralisch S, Lossner U, Bluher M, Paschke R, Stumvoll M \& Fasshauer M 2007 Growth hormone induces apelin mRNA expression and secretion in 
mouse 3T3-L1 adipocytes. Regulatory Peptides 139 84-89. (doi:10.1016/ j.regpep.2006.10.009)

Li RY, Song HD, Shi WJ, Hu SM, Yang YS, Tang JF, Chen MD \& Chen JL 2004 Galanin inhibits leptin expression and secretion in rat adipose tissue and 3T3-L1 adipocytes. Journal of Molecular Endocrinology 33 11-19. (doi:10.1677/jme.0.0330011)

Li P, Zhang XN, Pan CM, Sun F, Zhu DL, Song HD \& Chen MD 2011 Aldosterone perturbs adiponectin and PAI-1 expression and secretion in 3T3-L1 adipocytes. Hormone and Metabolic Research 43 464-469. (doi:10.1055/s-0031-1277226)

Lopez-Andres N, Inigo C, Gallego I, Diez J \& Fortuno MA 2008 Aldosterone induces cardiotrophin-1 expression in HL-1 adult cardiomyocytes. Endocrinology 149 4970-4978. (doi:10.1210/en.2008-0120)

Lorente-Cebrian S, Bustos M, Marti A, Martinez JA \& Moreno-Aliaga MJ 2010 Eicosapentaenoic acid up-regulates apelin secretion and gene expression in 3T3-L1 adipocytes. Molecular Nutrition \& Food Research 54 (Suppl 1) S104-S111. (doi:10.1002/mnfr.200900522)

Ma QY, Zhang XN, Jiang H, Wang ZQ, Zhang HJ, Xue LQ, Chen MD \& Song HD 2011 Mimecan in pituitary corticotroph cells may regulate ACTH secretion and the HPAA. Molecular and Cellular Endocrinology 341 71-77. (doi:10.1016/j.mce.2011.05.028)

Mazzucotelli A, Ribet C, Castan-Laurell I, Daviaud D, Guigne C, Langin D \& Valet P 2008 The transcriptional co-activator PGC- $1 \alpha$ up regulates apelin in human and mouse adipocytes. Regulatory Peptides 150 33-37. (doi:10.1016/j.regpep.2008.04.003)

Sonmez A, Celebi G, Erdem G, Tapan S, Genc H, Tasci I, Ercin CN, Dogru T, Kilic S, Uckaya G et al. 2010 Plasma apelin and ADMA Levels in patients with essential hypertension. Clinical and Experimental Hypertension 32 179-183. (doi:10.3109/10641960903254505)
Sowers JR, Whaley-Connell A \& Epstein M 2009 Narrative review: the emerging clinical implications of the role of aldosterone in the metabolic syndrome and resistant hypertension. Annals of Internal Medicine $\mathbf{1 5 0}$ 776-783. (doi:10.7326/0003-4819-150-11-200906020-00005)

Staermose S, Marwick TH, Gordon RD, Cowley D, Dowling A \& Stowasser M 2009 Elevated serum interleukin 6 levels in normotensive individuals with familial hyperaldosteronism type 1 . Hypertension 53 e31-e32. (doi:10.1161/HYPERTENSIONAHA.108. 128512)

Tatemoto K, Hosoya M, Habata Y, Fujii R, Kakegawa T, Zou MX, Kawamata Y, Fukusumi S, Hinuma S, Kitada C et al. 1998 Isolation and characterization of a novel endogenous peptide ligand for the human APJ receptor. Biochemical and Biophysical Research Communications 251 471-476. (doi:10.1006/bbrc.1998.9489)

Than A, Tee WT \& Chen P 2012 Apelin secretion and expression of apelin receptors in 3T3-L1 adipocytes are differentially regulated by angiotensin type 1 and type 2 receptors. Molecular and Cellular Endocrinology 351 296-305. (doi:10.1016/j.mce.2012.01.005)

Torpy DJ, Bornstein SR, Taylor W, Tauchnitz R \& Gordon RD 1999 Leptin levels are suppressed in primary aldosteronism. Hormone and Metabolic Research 31 533-536. (doi:10.1055/s-2007-978789)

Wehling M, Christ M \& Theisen K 1992 Membrane receptors for aldosterone: a novel pathway for mineralocorticoid action. American Journal of Physiology 263 E974-E979.

Wei L, Hou X \& Tatemoto K 2005 Regulation of apelin mRNA expression by insulin and glucocorticoids in mouse 3T3-L1 adipocytes. Regulatory Peptides 132 27-32. (doi:10.1016/j.regpep.2005.08.003)

Williams GH 2005 Aldosterone biosynthesis, regulation, and classical mechanism of action. Heart Failure Reviews 10 7-13. (doi:10.1007/ s10741-005-2343-3)

Received in final form 24 March 2013

Accepted 2 April 2013

Accepted Preprint published online 2 April 2013
(ㄷ) 2013 Society for Endocrinology Printed in Great Britain
Published by Bioscientifica Ltd. 